\title{
New Therapeutic Strategy for the Neurodegenerative Diseases: Pharmacological Modulation of $\mathrm{Ca}^{2+} /$ Camp Signaling Interaction
}

\author{
Afonso Caricati-Neto and Leandro Bueno Bergantin* \\ Department of Pharmacology, Federal University of Sao Paulo (UNIFESP), Brazil
}

*Corresponding author: Leandro Bueno Bergantin, Department of Pharmacology, Paulista School of Medicine, Laboratory of Autonomic and Cardiovascular Pharmacology, Federal University of Sao Paulo (UNIFESP), Rua Pedro de Toledo, 669 - Vila Clementino, São Paulo - SP, Postal Code: 04039-032, Brazil, Tel: 55-11-5576-4973, E-mail: leanbio39@yahoo.com.br

\begin{abstract}
Due to the involvement of the imbalance of neuronal $\mathrm{Ca}^{2+}$ homeostasis in the pathogenesis of several neurodegenerative diseases, the use of drugs to prevent or attenuate this imbalance emerged as a new therapeutic strategy for treating these diseases. Thus, our discovery of the involvement of the interaction between intracellular signaling pathways mediated by $\mathrm{Ca}^{2+}$ and CAMP $\left(\mathrm{Ca}^{2+} / \mathrm{cAMP}\right.$ signaling interaction) in the neurotransmission and neuroprotection, and its pharmacological modulation, could importantly contribute to the therapy of the neurodegenerative diseases, including Alzheimer's (AD) and Parkinson's (PD) Diseases. Then, in this review we discussed the fundamental findings of the $\mathrm{Ca}^{2+} / \mathrm{cAMP}$ signaling interaction, including its role in neurotransmission and neuroprotection, and its potential role as a new therapeutic target for the treatment of the neurodegenerative diseases.
\end{abstract}

\section{Keywords}

Neurodegenerative diseases, $\mathrm{Ca}^{2+} / \mathrm{cAMP}$ signaling interaction

\section{Introduction}

Several clinical studies performed since 1970's have reported that acute and chronic administration of L-type $\mathrm{Ca}^{2+}$ Channel Blockers (CCB) during antihypertensive therapy, such as nifedipine and verapamil, decreased arterial pressure but produced typical symptoms of sympathetic hyperactivity, such as tachycardia and enhance of catecholamine plasma levels [1]. Despite these adverse effects produced by CCB have been initially attributed to adjust reflex of arterial pressure and represented a potential risk for the antihypertensive therapy, the molecular mechanisms involved in these enigmatic effects remained unclear for decades.
Some in vitro studies performed since 1975 in isolated tissues richly innervated by sympathetic nerves (rodent vas deferens), to exclude the influence of adjusting reflex, showed that responses mediated by these nerves were completely inhibited by L-type CCB in concentrations higher than $1 \mu \mathrm{mol} / \mathrm{L}$, but unexpectedly and paradoxically potentiated in concentrations lower than $1 \mu \mathrm{mol} / \mathrm{L}$, characterizing CCB-induced sympathetic hyperactivity [2-4]. Defined by us as "calcium paradox" [5], these paradoxical CCB-effects remained unclear during almost forty years.

Using a classical model for in vitro study of sympathetic neurotransmission (rat vas deferens), we discovered in 2013 that this CCB-induced paradoxical sympathetic hyperactivity resulted from the increase of transmitter release from sympathetic neurons due to its modulatory action on the interaction between the intracellular signaling pathways mediated by $\mathrm{Ca}^{2+}$ and CAMP $\left(\mathrm{Ca}^{2+} / \mathrm{CAMP}\right.$ signaling interaction) in these neurons [5]. This discovery that solved the enigma of CCB-induced sympathetic hyperactivity was published in Cell Calcium [5].

The initial discovery of the role of $\mathrm{Ca}^{2+} / \mathrm{cAMP}$ signaling interaction in the neurotransmitter exocytosis and neurotransmission, and its pharmacological modulation, opens a large avenue to explore the participation of this intracellular signaling in other cellular responses. Our studies showed that the increment in neurotransmitter exocytosis produced by CCB (in low concentration) results from its modulatory action on the $\mathrm{Ca}^{2+} /$ cAMP signaling interaction [5]. This action results in

Citation: Caricati-Neto A, Bergantin LB (2018) New Therapeutic Strategy for the Neurodegenerative Diseases: Pharmacological Modulation of $\mathrm{Ca}^{2+} / \mathrm{Camp}$ Signaling Interaction. Int Arch Clin Pharmacol 4:015. doi.org/10.23937/2572-3987.1510015

Received: August 01, 2017: Accepted: May 07, 2018: Published: May 09, 2018

Copyright: (C) 2018 Caricati-Neto A, et al. This is an open-access article distributed under the terms of the Creative Commons Attribution License, which permits unrestricted use, distribution, and reproduction in any medium, provided the original author and source are credited. 
increment in the intracellular cAMP concentration $\left([\mathrm{cAMP}]_{\mathrm{c}}\right)$ that in turn stimulates the secretory machinery [5]. Interestingly, we concluded that this increment in $[\mathrm{cAMP}]_{\mathrm{c}}$ could stimulate other cellular responses, including cell survival [5-14]. Thus, we proposed that the pharmacological modulation of the $\mathrm{Ca}^{2+} / \mathrm{cAMP}$ signaling interaction could attenuate neuronal death resulting from cytosolic $\mathrm{Ca}^{2+}$ overload due to stimulation of cellular survival pathways mediated by Protein Kinase cAMP-dependent (PKA) and CAMP-Response Element Binding protein (CREB) (CAMP/PKA/CREB signaling pathway) [5-14].

Our discovery of the role of the $\mathrm{Ca}^{2+} / \mathrm{cAMP}$ signaling interaction in neurotransmission and neuroprotection, and its pharmacological modulation, may open a large avenue for the development of a new therapeutic strategy for neurodegenerative diseases [5-14]. We have proposed that this pharmacological modulation by use of the L-type CCB and compounds that increase the $[\mathrm{cAMP}]_{c^{\prime}}$, namely cAMP-enhancer compounds, could be effective in enhancing neurotransmission and neuroprotection in neurodegenerative diseases resulting from neurotransmission deficit and neuronal death, such as such as Alzheimer's (AD) and Parkinson's (PD) Diseases [5-14]. It is important to note that the growing increase in the life expectancy of the world's population has increased the concern about neurodegenerative diseases related to aging, such as AD and PD.

According to a 2015 United Nations report on world population ageing, the number of people aged 60 and older worldwide is projected to more than double in next 35 years, reaching almost 2.1 billion people. Most of this growth will come from developing regions of the world, although the oldest old, who are more than 80 years of age, are the fastest growing segment of the population in developed regions. Despite these improvements in life expectancy, $A D$ and $P D$, and related neurodegenerative conditions have arguably become the most dreaded maladies of older people. Then, in this review we will discuss how the pharmacological modulation of the $\mathrm{Ca}^{2+} / \mathrm{CAMP}$ signaling interaction could be a new therapeutic strategy to treat the neurodegenerative diseases, especially $A D$ and PD.

\section{Role of the $\mathrm{Ca}^{2+} /$ Camp Signaling Interaction in Neurotransmission}

Many experimental studies which have been initiated decades ago, using adrenal chromaffin cells as study model of exocytosis of secretory vesicles, established the notion of stimulus-secretion coupling to explain neurotransmitter release from central and peripheral neurons. In 1970's, it was demonstrated in adrenal chromaffin cells that the exocytosis of secretory vesicles containing transmitter (catecholamines, purines and other substances) is triggered by a rise in the cytosolic $\mathrm{Ca}^{2+}$ concentration $\left(\left[\mathrm{Ca}^{2+}\right]_{\mathrm{c}}\right)$ [15]. In 1990's, it was discovered a direct relationship between rise in $\left[\mathrm{Ca}^{2+}\right]_{c}$ and rapid transmitter release from adrenal chromaffin cells [16].

In addition to $\mathrm{Ca}^{2+}$, it was demonstrated that other intracellular messengers such as CAMP participate in the exocytosis of secretory vesicles containing transmitter. In 1988 , it was discovered that elevation of [CAMP] $]_{c}$ mediated by activation of Adenylyl Cyclases (AC) with forskolin enhanced exocytosis of secretory vesicles in adrenal chromaffin cells [17]. Although these evidences indicated that both $\mathrm{Ca}^{2+}$ and cAMP participate of transmitter exocytosis process in neurosecretory cells, the interaction between $\mathrm{Ca}^{2+}$ and cAMP signaling in this response remained unclear for decades.

In 2013, we discovered that neurotransmitter exocytosis from sympathetic neurons is finely regulated by interaction between intracellular signalling pathways mediated by $\mathrm{Ca}^{2+}$ and $\mathrm{CAMP}$, named $\mathrm{Ca}^{2+} / \mathrm{CAMP}$ signaling interaction [5]. Using isolated tissues richly innervated by sympathetic nerves (rat vas deferens) stimulated by electrical pulses, we discovered that neurogenic contractile responses of the smooth muscles of vas deferens were significantly reduced, or completely inhibited, by L-type CCB in concentrations more than $1 \mu \mathrm{mol} / \mathrm{L}$, but paradoxically increased in concentrations lower than $1 \mu \mathrm{mol} / \mathrm{L}$, characterizing CCB-induced sympathetic hyperactivity [5].

As the activity of $A C$ is finely regulated by $\mathrm{Ca}^{2+}$, the reduction of $\left[\mathrm{Ca}^{2+}\right]_{c}$ produced by L-type CCB results in increase of activity of $A C$, and consequent elevation of [cAMP] $]_{c}$ [5-14]. This elevation of [cAMP] results in stimulation of the cAMP-dependent Protein Kinase (PKA) that in turn activates Endoplasmic Reticulum (ER) $\mathrm{Ca}^{2+}$ channels, such as $\mathrm{ER}^{-\mathrm{Ca}^{2+}}$ channels regulated by Ryanodine Receptors (RyR), stimulating $\mathrm{Ca}^{2+}$ release and recruiting of secretory vesicles docked in plasma membrane, and thus increasing neurotransmitter release and synaptic concentration of neurotransmitters [5-14]. Then, we demonstrated that the reduction of $\mathrm{Ca}^{2+}$ influx through L-type Voltage-Activated $\mathrm{Ca}^{2+}$ Channels (VACC) produced by CCB increases synaptic transmission due to enhance of neurotransmitter release [5-14]. Our discovery solved the enigmatic "calcium paradox" of almost four decades involved in sympathetic hyperactivity produced by L-type CCB due to its modulatory action on the $\mathrm{Ca}^{2+} / \mathrm{cAMP}$ signaling interaction [5-14].

In addition, our studies also showed that combined use of the L-type CCB and CAMP-enhancer compounds, such as AC activators and phosphodiesterase (PDE) inhibitors, produced potentiation of sympathetic neurotransmission due to additional increase of exocytosis of secretory vesicles containing neurotransmitters (catecholamines, purines and others) [5-14]. We showed that the magnitude of contractile responses mediated by neurotransmitter released from sympathetic nerves by means Electrical Field Stimulation (EFS) in rat vas deferens (neurogenic contractions) were significantly re- 
(A)

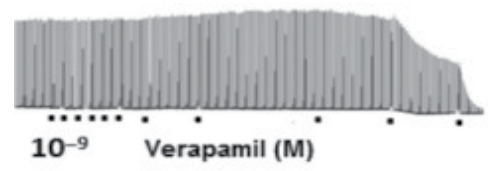

(C)

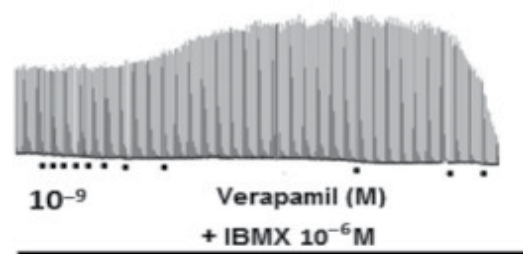

(B)
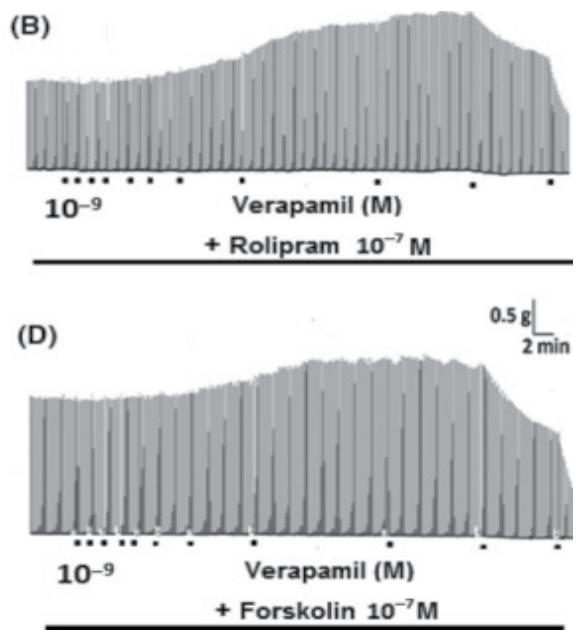

Figure 1: Increase of neurotransmission produced by pharmacological modulation of neural $\mathrm{Ca}^{2+} / \mathrm{cAMP}$ signaling interaction (A) Records showing that contractile responses mediated by neurotransmitter released from sympathetic nerves by means Electrical Field Stimulation (EFS) in rat vas deferens (neurogenic contractions) were significantly reduced by L-type CCB (verapamil) in high concentrations $\left(>10^{-6} \mathrm{M}\right)$, but paradoxically increased in concentrations below $10^{-6} \mathrm{M}$, characterizing CCB-induced sympathetic hyperactivity. This increase of neurogenic contractions by verapamil $\left(<10^{-6} \mathrm{M}\right)$ was potentiated by pre-treatment of isolated tissue with cAMP-enhancer compounds, such as rolipram $10^{-7} \mathrm{M}$; (B) IBMX $10^{-6} \mathrm{M}$; (C) and forskolin $10^{-7} \mathrm{M}$; (D) Each point below the record represents molar concentration of verapamil (interval of $0.5 \mathrm{log}$ unity). Each line below the record represents incubation time with cAMP-enhancer compounds. Representative records extracted from Bergantin, et al. [5].

duced by L-type CCB (verapamil) in high concentrations (> $1 \mu \mathrm{mol} / \mathrm{L})$, but paradoxically increased in concentrations below $1 \mu \mathrm{mol} / \mathrm{L}$, characterizing CCB-induced sympathetic hyperactivity (Figure 1A). These paradoxical CCB-effects were significantly potentiated by pre-treatment of vas deferens with CAMP-enhancer compounds, such as AC activators (forskolin) and Phosphodiesterase (PDE) inhibitors (rolipram and Isobutyl Methyl Xanthine (IBMX)) (Figure 1B, Figure 1C and Figure 1D). This potentiation by CAMP-enhancer compounds was prevented by inhibition of AC with SQ 22536.

These findings clearly demonstrated that the pharmacological modulation of neural $\mathrm{Ca}^{2+} / \mathrm{CAMP}$ signaling interaction enhances neurotransmitter release, causing significant increase of synaptic transmission [5-14]. On the basis of this finding, we postulated that the pharmacological modulation of this interaction could be a new strategy to increase neurotransmission in neurodegenerative diseases related to ageing, characterized by severe deficit in central neurotransmission, such as AD and PD [5-14].

\section{Role of the $\mathrm{Ca}^{2+} /$ Camp Signaling Interaction in Neuroprotection}

It is well known that an imbalance of intracellular $\mathrm{Ca}^{2+}$ homeostasis, especially cytosolic $\mathrm{Ca}^{2+}$ overload, contributes to the pathogenesis of neurodegenerative diseases related to aging, including $A D$ and PD [5-14]. Therefore, regulation of intracellular $\mathrm{Ca}^{2+}$ homeostasis may represent a new strategy for treatment of these diseases. As previously mentioned, blockade of the L-type VACC by CCB reduces $\mathrm{Ca}^{2+}$ influx resulting in attenuation of $\left[\mathrm{Ca}^{2+}\right]_{\mathrm{c}}$ and increase of $[\mathrm{CAMP}]_{\mathrm{c}}[5-14]$. We showed that this functional $\mathrm{Ca}^{2+} / \mathrm{CAMP}$ signaling interaction participates in several cellular responses, includ- ing neurotransmitter release [5-14]. Recently, it was showed that the treatment with L-type CCB reduces motor symptoms and attenuates progressive neuronal death in animal model of degenerative disease, suggesting that L-type CCB are potentially viable neuroprotective agents $[18,19]$. These findings reinforced the idea that attenuation of cytosolic $\mathrm{Ca}^{2+}$ overload produced by L-type CCB due to blockade of $\mathrm{Ca}^{2+}$ influx through L-type VACC could be an excellent pharmacological strategy to attenuate or prevent neuronal death in neurodegenerative diseases, such as PD and AD.

Some studies showed that increase of $[\mathrm{cAMP}]_{c}$ stimulates the survival cellular pathways mediated by CAMP/PKA/CREB signaling pathway [20-23]. In this way, we have proposed that the pharmacological modulation of the $\mathrm{Ca}^{2+} / \mathrm{CAMP}$ signaling interaction by combined use of L-type CCB and CAMP-enhancer compounds could stimulate neuroprotective response due to increase of $[\mathrm{cAMP}]_{\mathrm{c}}$ and attenuation of cytosolic $\mathrm{Ca}^{2+}$ overload [514]. In accordance to our studies, this pharmacological modulation could be a new neuroprotective therapeutic strategy to slow the progression of neurodegenerative diseases related to ageing, such as AD and PD. (Figure 2) shows how pharmacological modulation of the $\mathrm{Ca}^{2+} / \mathrm{CAMP}$ signaling interaction could produce increase of neurotransmission and neuroprotection in neurodegenerative diseases.

Pharmacological Modulation of Neural $\mathrm{Ca}^{2+} /$ Camp Signaling Interaction as a New Therapeutic Strategy for Treatment of Alzheimer's Disease (AD)

$A D$ is a progressive neurodegenerative disorder related to ageing characterized by cognitive and memory deterioration. Neuritic plaques represent the patho- 


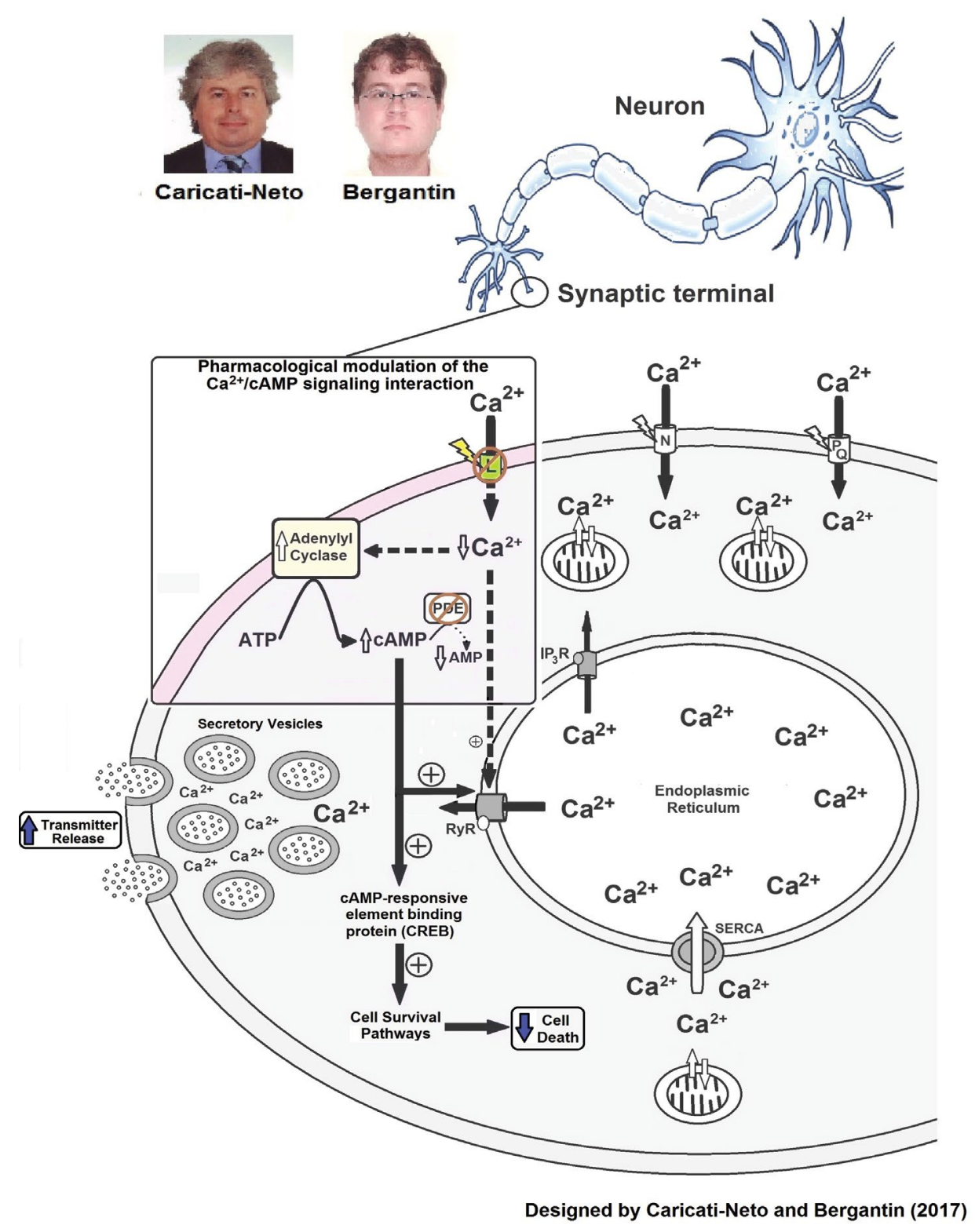

Figure 2: Increase of neurotransmitter release and attenuation of neuronal death (neuroprotection) produced by pharmacological modulation of the $\mathrm{Ca}^{2+} / \mathrm{CAMP}$ signaling interaction by combined use of L-type $\mathrm{Ca}^{2+}$ Channel Blockers (CCB) and cAMP-enhancer compounds. PDE - phosphodiesterase inhibitors. Figure extracted from Bergantin and Caricati-Neto [5-14].

logical status of $A D$ and are respectively related to the accumulation of the $\beta$-amyloid peptide $(A \beta)$ in brain tissues $[24,25]$. According to the amyloid hypothesis, the overproduction of $A \beta$ is a consequence of the disruption of homeostatic processes that regulate the proteolytic cleavage of the Amyloid Precursor Protein (APP). Genetic and age-related factors could contribute to a metabolic change, favoring the amyloidogenic processing of APP in detriment of the physiological secretory pathway $[24,25]$.

The neurotoxic potential of the $A \beta$ results from its biochemical properties that favor aggregation. These processes, along with a reduction of $A \beta$ clearance from the brain, lead to the extracellular accumulation of $A \beta$, and the subsequent activation of neurotoxic cascades that ultimately lead to cytoskeletal changes, neuronal dysfunction and cellular death [24]. Intracerebral amyloidosis development in AD patients is in an age-depen- dent manner, but recent evidences indicate that it may be observed in some subjects as early as in the third or fourth decades of life, with increasing magnitude in late middle age, and highest estimates in old age [24,25].

Therapies targeting the modification of amyloid-related cascades may be viewed as promising strategies to attenuate or even to prevent dementia [24]. Therefore, the cumulative knowledge on the pathogenesis of $A D$ derived from basic science models will hopefully be translated into clinical practice in the forthcoming years. Other targets relevant to $A D$ have also been considered in the last years for producing multitarget compounds $[26,27]$.

In addition to what has been discussed above, acetylcholinesterase (AChE) is another important target to treat the pathogenesis of $A D$ (cholinergic dysfunction hypothesis). Considering the current hypothesis of accumulation of the $A \beta$ in $A D$, this relies in the reduction 
of neurotransmitter acetylcholine (ACh) release in central cholinergic nervous system involved in cognitive function. Thus, the inhibition of ACh degradation by AChE is a potential target to treat AD [26-28].

An imbalance of intracellular $\mathrm{Ca}^{2+}$ homeostasis also contributes to the pathogenesis of AD [29]. Several evidences suggest that aging impairs ability of the brain to maintain intracellular $\mathrm{Ca}^{2+}$ homeostasis, which is likely to induce cellular damage due to cytosolic $\mathrm{Ca}^{2+}$ overload leading to neural death, and then resulting in cognitive dysfunction, such as AD [29]. Therefore, regulation of intracellular $\mathrm{Ca}^{2+}$ homeostasis may represent a new strategy for treatment of $A D$.

A 10-year follow-up study (2000 to 2010), involving 82,107 hypertensive patients of more than 60 years of age, showed that use of L-type CCB reduced blood pressure and risk of dementia in hypertensives, suggesting that these drugs could be clinically used to treat $A D$ [19]. Supportive findings for the neuroprotective effects of CCB have been showed in 1,241 elderly hypertensive patients with memory impairment [30]. The use of CCB decreased the risk of cognitive impairment and $A D$ independently of blood pressure levels when compared to patients not treated with CCB [30]. The long-term effects of antihypertensive therapy initiated with a long-acting dihydropiridine, such as nitrendipine, has been shown in the double-blind, placebo-controlled Syst-Eur trail in which the incidence of dementia was reduced by $55 \%$ [31].

Some studies have proposed that hybrid compounds having the moieties of tacrine, a potent inhibitor of AChE, and nimodipine, a L-type CCB could be useful to the AD pharmacotherapy $[26,27]$. In addition, galantamine (a moderate AChE inhibitor and a potent allosteric ligand of nicotinic cholinoceptors) has been used to improve cognition and behavior in patients with $A D$ [28]. Studies using AD model rats showed that CAMP-enhancer compounds, such as nobiletin (a polymethoxylated flavone from citrus peels) and oxyntomodulin (a proglucagon-derived peptide that co-activates the GLP1 receptor and the glucagon receptor) produce neuroprotective effects mediated by intracellular CAMP synthesis, activation of PKA and MAPK pathways and phosphorylation of CREB $[21,23]$.

Our discovery of the involvement of the $\mathrm{Ca}^{2+} / \mathrm{cAMP}$ signaling interaction in the neurotransmission and neuroprotection has produced important advances in the understanding of the pathophysiology and pharmacology of AD [5-14]. These advances allowed us to propose that pharmacological modulation of the $\mathrm{Ca}^{2+} / \mathrm{CAMP}$ signaling interaction produced by combined use of the L-type CCB (used in the antihypertensive therapy), such as isradipine, and CAMP-enhancer compounds (used in the anti-depressive therapy), such as rolipram, could represent a new therapeutic strategy for treatment of $A D$ in humans. This pharmacological modulation could attenuate cognitive deficit due to increase in central cholinergic neurotransmission caused by increment in ACh release from cholinergic neurons [5-14]. In addition, the pharmacological modulation of the $\mathrm{Ca}^{2+} / \mathrm{CAMP}$ signaling interaction could reduce or prevent neuronal death caused by cytosolic $\mathrm{Ca}^{2+}$ overload due to increase of [CAMP $]_{c}$ and stimulation of cellular survival pathways mediated by CAMP/PKA/CREB signaling pathway [2023]. Thus, we have proposed that the pharmacological modulation of this signaling could be a new therapeutic strategy to slow the progression and reduce the symptoms of $A D$ [5-14].

Pharmacological Modulation of Neural $\mathrm{Ca}^{2+} /$ Camp Signalling Interaction as a New Therapeutic Strategy for Treatment of Parkinson's Disease (PD)

$\mathrm{PD}$ is an age-associated progressive neurodegenerative disorder that has gained crescent social and economic impact due to the aging of the western society. While the distinctive motor symptoms of PD have been described for centuries, cognitive impairment has only recently been recognized as a central feature. All current therapies are symptomatic and fail to reverse or halt the progression of dopaminergic neurons loss. PD mainly results from dopamine loss in the substantia nigra, which results in reduction of dopamine release from striatal dopaminergic neurons due to neuronal death $[32,33]$. These central dopaminergic dysfunctions result in the recognizable signs of asymmetrical bradykinesia and hypokinesia (slowness and reduced amplitude of movement), muscle rigidity (stiffness) and rest tremor, consequences from modifying motor control $[32,33]$. Rest tremor, prominent asymmetry and a good response to levodopa are the features that most accurately predict PD pathology [32,33].

Functional imaging of the dopaminergic system using cerebral single photon emission computed tomography or positron emission tomography can be useful in diagnosis of early PD. Positron Emission Tomography (PET) studies examining the rate of decline in dopamine-producing cells suggest that humans have already lost $50 \%-70 \%$ of their nigral neurons, before they develop motor symptoms, and it has been estimated that the duration of this "presymptomatic" phase is about 5 years [32,33]. Early diagnoses will become a critical issue if effective neuroprotective drugs become available. In fact, increasing dopamine, mainly using the Levodopa combined with a dopa-decarboxylase inhibitor remains the most potent drug therapy for reversing motor impairment. A higher maintenance dose of Levodopa (eg, $200 \mathrm{mg}$ three times daily compared with an initial dose of $100 \mathrm{mg}$ three times daily) provides slightly greater benefit for reducing motor symptoms, but at the cost of earlier wearing-off symptoms and dyskinesias $[32,33]$. The combination of novel concepts may lead to advances in PD research with the promise of finding 
compounds that are both effective, and fast-acting, including in patients who have tried other therapies with limited success.

Several lines of evidences suggest that an imbalance of intracellular $\mathrm{Ca}^{2+}$ homeostasis contributes to the pathogenesis of PD. Thus, regulation of intracellular $\mathrm{Ca}^{2+}$ homeostasis may represent a new strategy for treatment of PD. Several studies suggest that aging impairs ability of the brain to maintain intracellular $\mathrm{Ca}^{2+}$ homeostasis, which is likely to induce cellular damage leading to death of dopaminergic neurons in the substantia nigra, and resulting in motor dysfunction due to reduction of dopamine release in striatal dopaminergic neurons [18,34].

A phase II clinical study published in 2016 showed that treatment with L-type CCB isradipine was safely tolerated to reduce motor symptoms in PD patients [34]. In addition, it was shown that the treatment with isradipine reduces motor symptoms and attenuates progressive death of dopamine neurons from substantia nigra in animal model of PD [18]. This study showed that the isradipine produced a dose-dependent sparing of dopaminergic fibers and cell bodies at concentrations achievable in humans [18], suggesting that L-type CCB are potentially viable neuroprotective agents useful for $P D$ treatment. These finding reinforced the idea that attenuation of cytosolic $\mathrm{Ca}^{2+}$ overload produced by L-type CCB due to blockade of $\mathrm{Ca}^{2+}$ influx through L-type VACC could be an excellent pharmacological strategy to attenuate or prevent the death of dopaminergic neurons in PD.

Our discovery of the involvement of the $\mathrm{Ca}^{2+} / \mathrm{cAMP}$ signaling interaction in the neurotransmission and neuroprotection has contributed to understanding of the pathophysiology and pharmacology of PD [5-14]. These advances allowed us to propose that pharmacological modulation of the $\mathrm{Ca}^{2+} / \mathrm{cAMP}$ signaling interaction produced by combined use of the L-type CCB and CAMP-enhancer compounds could represent a new therapeutic strategy for treatment of human PD. We believe that pharmacological modulation of the $\mathrm{Ca}^{2+} / \mathrm{cAMP}$ signaling interaction could attenuate dopamine release deficit, increasing central dopaminergic neurotransmission involved in the control of motor function. In addition, pharmacological modulation of this interaction could contribute to reduce death of dopaminergic neurons due to attenuation of cytosolic $\mathrm{Ca}^{2+}$ overload, increase of [CAMP] and stimulation of cellular survival regulated by AMP/PKA/CREB signaling pathway [20-23]. Thus, pharmacological modulation of $\mathrm{Ca}^{2+} / \mathrm{CAMP}$ signaling interaction could be a new therapeutic strategy to slow the progression of PD [5-14].

\section{Conclusion}

Our recent discovery of the $\mathrm{Ca}^{2+} / \mathrm{CAMP}$ signaling interaction could promote important advances in the pathophysiology and pharmacology of the neurodegen- erative diseases related to ageing. These advances can contribute to drug development more effective and safer to attenuate or prevent clinical symptoms of neurodegenerative diseases, such as AD and PD.

\section{Disclosure Statement}

Caricati-Neto and Bergantin thank the continued financial support from CAPES, CNPq and FAPESP (Bergantin's Postdoctoral Fellowship FAPESP \#2014/102743). The authors also thank Elsevier - "author use": Reuse of portions or extracts from the article in other works - https://www.elsevier.com/_data/assets/pdf_ file/0007/55654/AuthorUserRights.pdf.

\section{References}

1. Grossman E, Messerli FH (1998) Effect of calcium antagonists on sympathetic activity. Eur Heart J 19: 27-31.

2. Kreye VA, Lüth JB (1975) Proceedings: Verapamil-induced phasic contractions of the isolated rat vas deferens. Naunyn Schmiedebergs Arch Pharmacol 287: 43.

3. French AM, Scott NC (1981) A comparison of the effects of nifedipine and verapamil on rat vas deferens. $\mathrm{Br} \mathrm{J}$ Pharmacol 73: 321-323.

4. Moritoki H, Iwamoto $T$, Kanaya J, Maeshiba $Y$, Ishida $Y$, et al. (1987) Verapamil enhances the non-adrenergic twitch response of rat vas deferens. Eur J Pharmacol 140: 75-83.

5. Bergantin LB, Souza CF, Ferreira RM, Smaili SS, Jurkiewicz NH, et al. (2013) Novel model for "calcium paradox" in sympathetic transmission of smooth muscles: role of cyclic AMP pathway. Cell Calcium 54: 202-212.

6. Caricati-Neto A, García AG, Bergantin LB (2015) Pharmacological implications of the $\mathrm{Ca}^{2+} / \mathrm{cAMP}$ signalling interaction: from risk for antihypertensive therapy to potential beneficial for neurological and psychiatric disorders. Pharmacol Res Perspect 3: e00181.

7. Bergantin LB, Jurkiewicz A, García AG, Caricati-Neto A (2015) A Calcium Paradox in the Context of Neurotransmission. J Pharmac Pharmacol 3: 253-261.

8. Bergantin LB, Caricati-Neto A (2016) Challenges for the pharmacological treatment of neurological and psychiatric disorders: Implications of the $\mathrm{Ca}^{2+} / \mathrm{cAMP}$ intracellular signalling interaction. Eur J Pharmacol 788: 255-260.

9. Bergantin LB, Caricati-Neto A (2016) Insight from "Calcium Paradox" due to $\mathrm{Ca}^{2+} / \mathrm{cAMP}$ Interaction: Novel Pharmacological Strategies for the Treatment of Depression. Int Arch Clin Pharmacol 2: 007.

10. Bergantin LB, Caricati-Neto A (2016) Novel Insights for Therapy of Parkinson's disease: Pharmacological Modulation of the $\mathrm{Ca}^{2+} / \mathrm{cAMP}$ Signalling Interaction. Austin Neurol Neurosci 1: 1009.

11. Bergantin LB, Caricati-Neto A (2016) Recent advances in pharmacotherapy of neurological and psychiatric disorders promoted by discovery of the role of $\mathrm{Ca}^{2+} / \mathrm{CAMP}$ signaling interaction in the neurotransmission and neuroprotection. Adv Pharmac J 1: 66-70.

12. Bergantin LB, Caricati-Neto A (2016) From discovering "calcium paradox" to $\mathrm{Ca}^{2+} / \mathrm{CAMP}$ interaction: Impact in human health and disease. Scholars Press 120.

13. Bergantin LB, Caricati-Neto A (2016) Impact of interaction of $\mathrm{Ca}^{2+} / \mathrm{cAMP}$ Intracellular Signalling Pathways in Clinical 
Pharmacology and Translational Medicine. Clinical Pharmacology and Translational Medicine 1-4.

14. Bergantin LB, Caricati-Neto A (2016) Challenges for the Pharmacological Treatment of Dementia: Implications of the $\mathrm{Ca}^{2+} / \mathrm{cAMP}$ Intracellular Signalling Interaction. Eur J Pharmacol 788: 255-260.

15. Baker PF, Knight DE (1978) Calcium-dependent exocytosis in bovine adrenal medullary cells with leaky plasma membranes. Nature 276: 620-622.

16. Neher E, Zucker RS (1993) Multiple calcium-dependent processes related to secretion in bovine chromaffin cells. Neuron 10: 21-30.

17. Chern YJ, Kim KT, Slakey LL, Westhead EW (1988) Adenosine receptors activate adenylate cyclase and enhance secretion from bovine adrenal chromaffin cells in the presence of forskolin. J Neurochem 50: 1484-1493.

18. Ilijic E, Guzman JN, Surmeier DJ (2011) The L-type channel antagonist isradipine is neuroprotective in a mouse model of Parkinson's disease. Neurobiol Dis 43: 364-371.

19. Wu CL, Wen SH (2016) A 10-year follow-up study of the association between calcium channel blocker use and the risk of dementia in elderly hypertensive patients. Medicine (Baltimore) 95: e4593.

20. Sommer N, Loschmann PA, Northoff GH, Weller M, Steinbrecher A, et al. (1995) The antidepressant rolipram suppresses cytokine production and prevents autoimmune encephalomyelitis. Nat Med 1: 244-248.

21. Onozuka H, Nakajima A, Matsuzaki K, Shin RW, Ogino K, et al. (2008) Nobiletin, a citrus flavonoid, improves memory impairment and Abeta pathology in a transgenic mouse model of Alzheimer's disease. J Pharmacol Exp Ther 326: 739-744.

22. Xiao L, O'Callaghan JP, O'Donnell JM (2011) Effects of repeated treatment with phosphodiesterase-4 inhibitors on cAMP signaling, hippocampal cell proliferation, and behavior in the forced-swim test. J Pharmacol Exp Ther 338: 641-647.

23. Li Y, Wu KJ, Yu SJ, Tamargo IA, Wang Y, et al. (2017) Neurotrophic and neuroprotective effects of oxyntomodulin in neuronal cells and a rat model of stroke. Exp Neurol 288: 104-113.
24. De-Paula VJ, Radanovic M, Diniz BS, Forlenza OV (2012) Alzheimer's disease. Subcell Biochem 65: 329-352.

25. Mohamed T, Shakeri A, Rao PP (2016) Amyloid cascade in Alzheimer's disease: Recent advances in medicinal chemistry. Eur J Med Chem 113: 258-272.

26. Maroto M, de Diego AM, Albiñana E, Fernandez-Morales JC, Caricati-Neto A, et al. (2011) Multi-target novel neuroprotective compound ITH33/IQM9.21 inhibits calcium entry, calcium signals and exocytosis. Cell Calcium 50: 359-369.

27. Ismaili L, Refouvelet B, Benchekroun M, Brogi S, Brindisi M (2016) Multitarget compounds bearing tacrine- and donepezil-like structural and functional motifs for the potential treatment of Alzheimer's disease. Prog Neurobiol 151: 4-34.

28. Samochocki M, Höffle A, Fehrenbacher A, Jostock R, Ludwig J, et al. (2003) Galantamine is an allosterically potentiating ligand of neuronal nicotinic but not of muscarinic acetylcholine receptors. J Pharmacol Exp Ther 305: 10241036.

29. Kawamoto EM, Vivar C, Camandola S (2012) Physiology and pathology of calcium signaling in the brain. Front Pharmacol 3: 61.

30. Hanon O, Pequignot R, Seux ML, Lenoir H, Bune A, et al. (2006) Relationship between antihypertensive drug therapy and cognitive function in elderly hypertensive patients with memory complaints. J Hypertens 24: 2101-2107.

31. Forette F, Seux ML, Staessen JA, Thijs L, Babarskiene MR, et al. (2002) Systolic Hypertension in Europe Investigators The prevention of dementia with antihypertensive treatment: new evidence from the systolic hypertension in Europe (Syst-Eur) study. Arch Intern Med 162: 2046-2052.

32. Hayes MW, Fung VS, Kimber TE, O'Sullivan JD (2010) Current concepts in the management of Parkinson disease. Med J Aust 192: 144-149.

33. Salat D, Noyce AJ, Schrag A, Tolosa E (2016) Challenges of modifying disease progression in prediagnostic Parkinson's disease. Lancet Neurol 15: 637-648.

34. Swart T, Hurley MJ (2016) Calcium channel antagonists as disease-modifying therapy for Parkinson's disease: Therapeutic rationale and current status. CNS Drugs 30: 11271135. 